This item was submitted to Loughborough's Research Repository by the author.

Items in Figshare are protected by copyright, with all rights reserved, unless otherwise indicated.

\title{
Energetic impact of size-selected metal cluster ions on graphite
}

PLEASE CITE THE PUBLISHED VERSION

PUBLISHER

(C) American Physical Society

LICENCE

CC BY-NC-ND 4.0

\section{REPOSITORY RECORD}

Carroll, S.J., S.G. Hall, R.E. Palmer, and Roger Smith. 2019. "Energetic Impact of Size-selected Metal Cluster Ions on Graphite". figshare. https://hdl.handle.net/2134/1733. 


\title{
Energetic Impact of Size-Selected Metal Cluster Ions on Graphite
}

\author{
S. J. Carroll, S. G. Hall, and R. E. Palmer \\ Nanoscale Physics Research Laboratory, School of Physics and Astronomy, The University of Birmingham, Edgbaston, \\ Birmingham B15 2TT, United Kingdom \\ R. Smith \\ Department of Mathematical Sciences, Loughborough University, Loughborough, Leicestershire LE11 3TU, United Kingdom
} (Received 23 March 1998)

\begin{abstract}
We have investigated the impact of size-selected metal cluster ions $\left(\mathrm{Ag}_{n}{ }^{-}\right)$on a covalently bonded substrate (graphite) over the energy range $15-1500 \mathrm{eV}$ by a combination of scanning tunneling microscopy and molecular dynamics simulations. The key result is that the fate of the cluster (penetration into the surface versus diffusion and aggregation on the surface), at intermediate energies, depends on the lateral localization of the cluster kinetic energy at specific surface sites and thus, for small clusters, on the orientation of the cluster and the target substrate site. [S0031-9007(98)07519-X]
\end{abstract}

PACS numbers: 79.20.Rf, 61.46.+w, 68.55.Ln, 81.05.Ys

The rapidly growing interest in the deposition of sizeselected atomic clusters on surfaces is motivated both by technology and by basic physical questions [1,2]. From the technological viewpoint, clusters can be regarded as the precursors to a new generation of nanostructured materials and devices [3]. From a fundamental perspective, deposition allows the whole range of surface physics techniques to be deployed to explore the properties of relatively numerous confined quantum systems [4]. The dynamics of the cluster-surface interaction is itself the subject of increasing attention [5-7]. Recent highlights include the identification of a new mechanism (Brownian motion) for the (exceptionally) fast diffusion of metal clusters over the surface of graphite [5,8], and experimental verification [9] of the predicted [10] "soft landing" scheme in which an incident metal cluster is slowed down by a "breaking layer" of rare gas on the surface. This new generation of experiments has been accompanied by increasingly sophisticated atomistic simulations [10], especially in the case of metal clusters on metal surfaces [11-14], where similar potentials may be employed for both the cluster and surface.

In this Letter we explore, through a combination of scanning tunneling microscopy (STM) experiments and molecular dynamics simulations, the interaction of ionized, size-selected metal (Ag) clusters with (covalently bonded) graphite over a wide range of impact energies $(15-1500 \mathrm{eV})$. While graphite is a natural (and popular) substrate for cluster deposition and diffusion experiments [5,15-17], the impact of metal clusters on graphite has not previously been modeled. However, recent experiments $[18,19]$ and simulations [19-24] concerned with the impact of energetic (atomic and cluster) ions on this surface provide an invaluable reference point for interpreting the novel features of the cluster-surface interaction. In particular, we show that the fate of the impacting cluster depends on the lateral localization ("focusing") of the cluster kinetic energy at specific atomic sites of the sur- face, which determines whether the cluster penetrates the surface via displacement of a (single) surface carbon atom. The defining cluster geometric parameter which emerges is the "footprint" on the surface, which for small clusters depends on the orientation with respect to the surface plane.

The molecular dynamics (MD) simulations have to find a way of connecting two quite different types of (empirical) potentials describing the metal cluster and the covalently bonded graphite substrate. The graphite substrate was described using the $N$-body Brenner [25] potential, which gives the correct cohesive energy for carbon in the graphite and diamond structures. We simulated the $\mathrm{Ag}-\mathrm{Ag}$ potential in the cluster using the many body potential obtained via the embedded atom method by Ackland et al. [26]. The $\mathrm{Ag}_{3}$ cluster was arranged as a linear chain, with minimum energy atomic separations as calculated by Bonačić-Koutecký [27], to follow the experimental situation as closely as possible. The interaction between the $\mathrm{C}$ and $\mathrm{Ag}$ atoms requires some improvization. We employed the empirical two body potential obtained by Rafii-Tabar [28] et al. which produces an accurate simulation of the STM results for the adsorption of $\mathrm{Ag}$ atoms and clusters on the graphite surface. Simulations were performed on an $18 \times 18 \mathrm{~nm}$ graphite lattice, initially $0 \mathrm{~K}$. Fixed boundaries were employed. The simulation ran for $20 \mathrm{ps,} \mathrm{with} \mathrm{a}$ time step of $0.75 \mathrm{fs}$. All the images were taken at a simulation time of $20 \mathrm{ps}$. We present here the main themes emerging from the MD investigations, illustrated by images of typical results.

The experimental investigations were performed with a cluster-ion source based on caesium sputtering of the (Ag) target. The source is specifically designed for low energy deposition of size-selected clusters [29]. The clusters, in this case $\mathrm{Ag}_{3}{ }^{-}$and $\mathrm{Ag}_{5}{ }^{-}$, were projected at normal incidence onto the graphite substrate (10 minutes after insertion at a pressure of $10^{-7} \mathrm{mbar}$ ). The graphite samples were prepared by cleaving with Scotch tape. The samples were subsequently studied in air with a 
commercial STM (DME Rasterscope 4000) operated in the constant current mode. The STM tips were made from mechanically cut Pt-Ir wire. Typical experimental conditions used were a tunneling current of $0.2 \mathrm{nA}$ and a tip bias of $250 \mathrm{mV}$. The system $\mathrm{Ag}_{n} /$ graphite is attractive as our STM investigations have found this is system stable against morphological changes over a period of weeks. Further, our high resolution transmission electron microscopy studies have shown no evidence for the contamination of nanometer-scale Ag clusters deposited on amorphous carbon.

The principal experimental effect of varying the kinetic energy of the deposited $\mathrm{Ag}_{n}$ clusters, for a given dose of $\mathrm{Ag}_{3}{ }^{-}$clusters $\left(\sim 6 \times 10^{13}\right.$ ions per $\left.\mathrm{cm}^{2}\right)$ is demonstrated in Fig. 1. As the energy is increased from $15 \mathrm{eV}$ (a), through $150 \mathrm{eV}$ (b), to $300 \mathrm{eV}$ (c), a corresponding increase in feature density is immediately apparent. This increase is quantified in Fig. 2(a), which is a plot of the ratio of the number density of surface features to the number density of incident clusters. As the deposition energy of the clusters increases, the number of surface features per incident cluster tends to unity. Since the features on the surface are typically a few nanometers in diameter it is natural to conclude, in harmony with previous work, that at low deposition energies the clusters diffuse over the surface and aggregate to form large islands. The behavior at high energies is clearly quite different. The atomic displacement energy for graphite is $34 \mathrm{eV}$ [30]. Thus, while penetration by clusters at $15 \mathrm{eV}$ is unlikely — as confirmed by the evidence for diffusion and aggregation - it is reasonable to conclude that the majority of clusters deposited at $300 \mathrm{eV}$ will penetrate the surface layer. Indeed, previous studies [19] of the energetic impact of atomic ions on graphite have established the nature of the surface features, or "bumps," caused by particle penetration into the surface. Basically, penetration of the projectile produces interstitials or vacancies, and the bumps observed arise from plastic deformation of the surface layer above such defects $[19,21,22]$. Thus we can explain the one-to-one ratio between the number of surface features and the number of deposited clusters at high cluster impact energy by the creation of one surface bump for each cluster which penetrates the surface layer.

A further experimental test of the fate of the deposited cluster is provided by considering the morphology in the vicinity of a surface step, Figs. 2(b), 2(c). From the lower coordination number of the carbon atoms at the step it is expected that the steps have a higher chemical activity than the basal plane, leading to preferential nucleation of mobile species along the steps and a zone of depleted island density about the step. This behavior has been noted for both $\mathrm{Ag}$ atoms and $\mathrm{Ag}_{400}$ clusters on graphite [16,17]. Figure 2(b) shows the behavior near a step for the deposition of $\mathrm{Ag}_{3}{ }^{-}$cluster ions at $150 \mathrm{eV}$ [cf. Fig. 1(b)]. In Fig. 2(b) we see that larger $(\sim 6 \mathrm{~nm})$ particles have collected at the step and are consequently absent from the surrounding terrace, suggesting they are mobile surface islands. Smaller $(\sim 3 \mathrm{~nm})$ particles show no number density dependence on their proximity to the step, revealing that they are immobile surface bumps. Thus, at this intermediate energy, there is a mixture of cluster penetration (giving bumps) and cluster diffusion (giving mobile islands). The result of high energy $(1500 \mathrm{eV})$ deposition of $\mathrm{Ag}_{5}{ }^{-}$clusters can be seen in Fig. 2(c). In this case the only features observed are bumps, produced by cluster impact, which show no tendency to collect at surface steps.

The biggest challenge for the molecular dynamics simulations of the cluster-surface interaction is probably to explain the "mixed" behavior at intermediate energies, e.g., $150 \mathrm{eV}$. Any notion that the fate of the cluster is dependent simply on the kinetic energy and size of the cluster cannot account for two different outcomes (i.e., implantation and diffusion) for a given energy and size. Figure 3 shows the result of MD simulations of the impact of $\mathrm{Ag}_{3}$ clusters at (a) $15 \mathrm{eV}$ and (b) $300 \mathrm{eV}$. The relative cluster-surface orientation and impact site are shown in the inset for each case. For all the trajectories considered, deposition at $15 \mathrm{eV}$ always resulted in an intact $\mathrm{Ag}_{3}$ cluster absorbed on the surface, Fig. 3(a) (though sometimes the cluster adopted a linear configuration). At room temperature we would expect these adsorbed clusters to diffuse over the surface and aggregate to form the $\sim 6 \mathrm{~nm}$ islands seen in Fig. 1(a); the time scale for diffusion is currently way beyond the scope of an atomistic MD simulation. Impact of $\mathrm{Ag}_{3}$ clusters at high energy $(300 \mathrm{eV})$ is illustrated by the cross-sectional view of Fig. 3(b). The cluster penetrates below the graphite surface layer, leaving an amorphous trail which joins together the individual covalently bonded graphite layers.
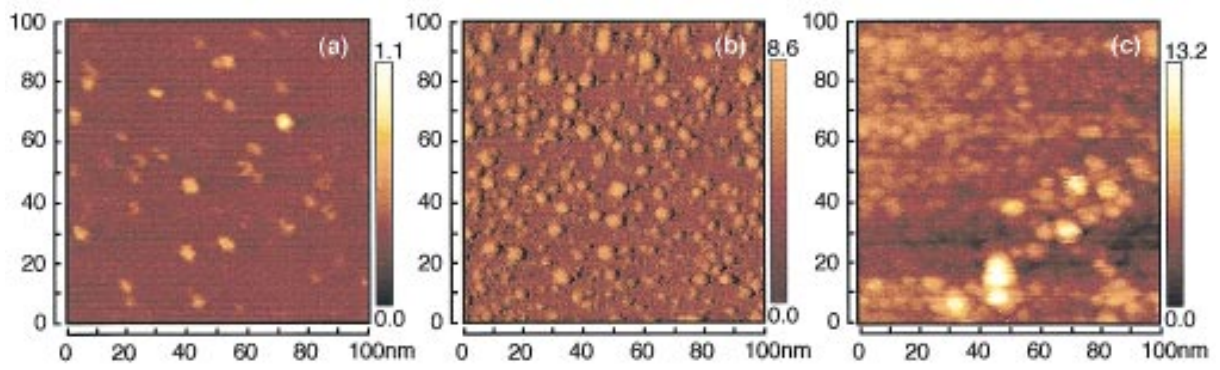

FIG. 1(color). STM images after the impact of $\mathrm{Ag}_{3}{ }^{-}$clusters on graphite at (a) $15 \mathrm{eV}$, (b) $150 \mathrm{eV}$, and (c) $300 \mathrm{eV}$. 

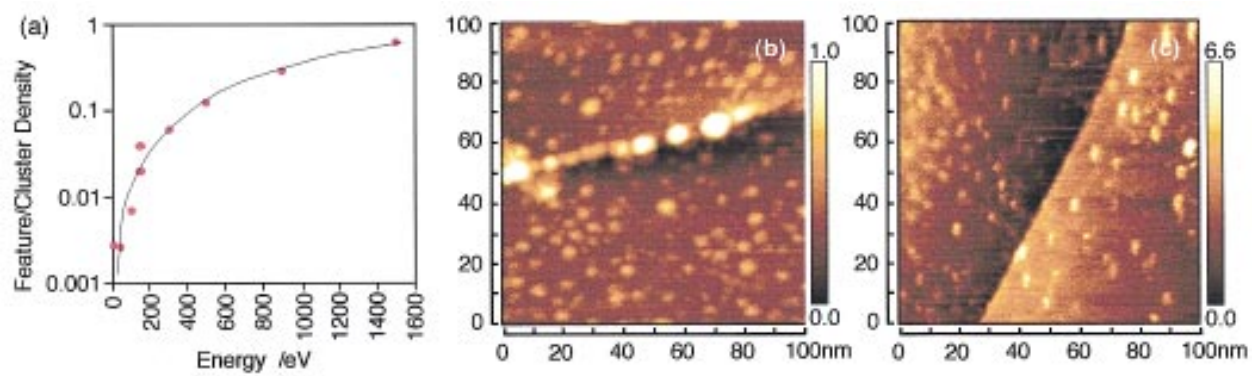

FIG. 2(color). (a) Plot of the surface feature density versus the deposited cluster density. STM images after the deposition of (b) $\mathrm{Ag}_{3}{ }^{-}$clusters on graphite at $150 \mathrm{eV}$, and (c) $\mathrm{Ag}_{5}{ }^{-}$at $1500 \mathrm{eV}$.

Cluster penetration to the $3 \mathrm{rd}-5$ th layer is normal at this energy, dependent on the impact parameters. When the cluster penetrates the surface a bump is usually formed close to the impact site; such a bump can be seen in the magnified $z$ scale image in Fig. 3(c). In this case the $\mathrm{Ag}_{3}$ cluster was oriented parallel to the surface and impacted at $150 \mathrm{eV}$, resulting in partial implantation of the cluster together with the formation of a bump, arising from the local distortion of the graphite surface after cluster impact.

The critical case of cluster impact at intermediate energies (e.g., $150 \mathrm{eV}$ ) is addressed in Fig. 4. We find that the morphology resulting from cluster impact depends strongly on the orientation of the (linear) $\mathrm{Ag}_{3}$ cluster with respect to the surface plane as well as impact site. For the configuration shown in Fig. 4(a), where the cluster impacts directly onto an $\alpha$ site, all the cluster atoms penetrate into the second graphite layer. When the cluster is oriented parallel to the surface, Fig. 4(b), partial penetration of the cluster is noted. If the cluster is oriented normal to the surface, and incident on a hole site, the cluster does not penetrate the graphite surface, and is simply adsorbed [Fig. 4(c)]. In the experiment both the cluster orientation and the impact site are random. Thus some penetration (and hence bump formation) is observed, while cluster atoms remaining on the surface after the impact are able to diffuse and aggregate to form the islands observed. The cluster's behavior can be understood in terms of the atomic displacement energy of the graphite atoms (previously noted to be $34 \mathrm{eV}$ [30]. In case (a) a significant amount of the cluster kinetic energy $(150 \mathrm{eV})$ is focused onto a single carbon atom at the $\alpha$ site. Consequently this atom is punched from the surface into the bulk leaving a gap through which the cluster can enter the surface. By arranging the cluster parallel to the surface [case (b)], the number of surface atoms with which the cluster interacts is increased, so the kinetic energy of $150 \mathrm{eV}$ is spread out and penetration is more shallow. When the cluster hits the hole site [case (c)], the energy is spread out over all six atoms around the hole, and no penetration into the surface occurs because no surface atoms are displaced. Thus, when considering the outcome of cluster deposition, it is not just the energy (or even energy per atom) of the incident cluster which is important, but also the local area of the surface with which the cluster interacts. The cluster's energy must be focused into a small area for penetration or implantation to occur via displacement of surface atoms. For larger clusters the orientation of the cluster and the impact site must become less important, as the increased cluster size effectively averages out these effects.

Finally, we emphasize that the particular trajectories simulated here are simply examples of the kind of trajectories over which the experiment averages; indeed, in practice, the cluster will probably be vibrationally excited as well. (Recent work has suggested this vibrational excitation could be large, up to $\sim 1 \mathrm{eV}$ [31]). The vibrational (and rotational) motion of the clusters atoms will introduce further smearing or averaging over the impact parameters. The key point is that the simulations do illustrate a range of possible outcomes, notably at intermediate energies, consistent with the mixture of diffusion and implantation deduced from the experiment. (a)

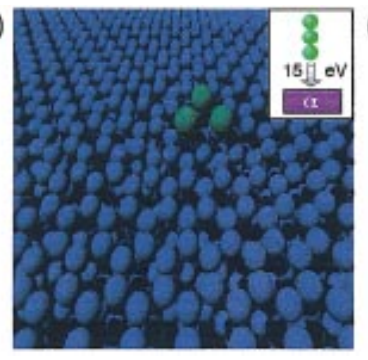

(b)

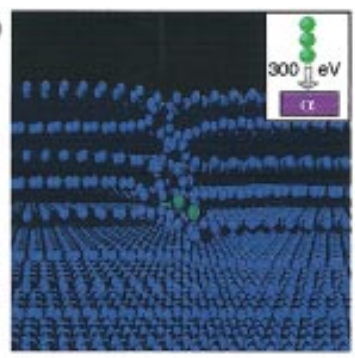

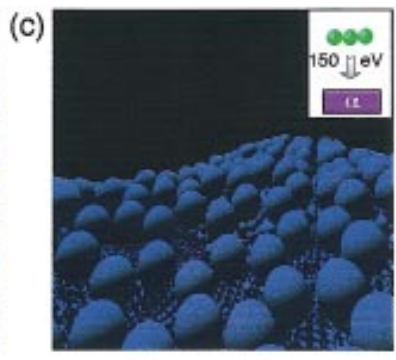

FIG. 3(color). MD simulation of the impact of $\mathrm{Ag}_{3}$ clusters; see inset to each image for the impact parameters. Note that the vertical scale in (c) is magnified by $5 \times$. 
(a)

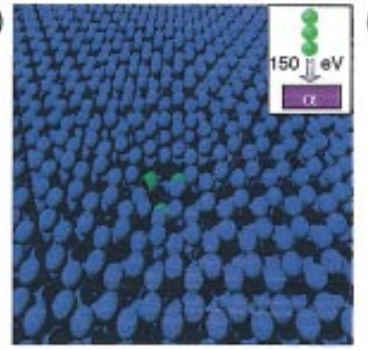

(b)

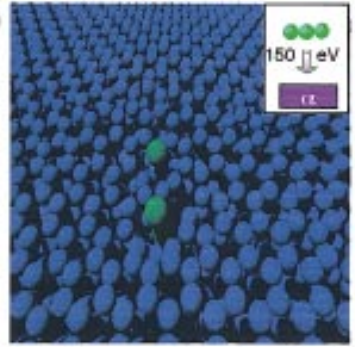

(c)

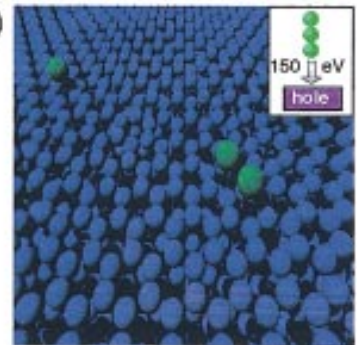

FIG. 4(color). MD simulation of $\mathrm{Ag}_{3}$ impact at intermediate energy $(150 \mathrm{eV})$; see inset in each image for detailed impact parameters.

In summary, the combination of experiment and molecular dynamics modeling provides a consistent framework for understanding the impact of metal clusters upon the surface of a covalently bonded material, graphite. Our work highlights the need to consider the area of the surface (footprint) and the specific impact parameters (cluster orientation, surface atomic site) associated with the clustersurface interaction. In particular, penetration of the cluster into the surface requires that sufficient cluster kinetic energy is focused into a single surface atom. Similar effects are to be anticipated in the energetic interaction of any cluster with the surface of a covalently bonded material, and will also control the morphology, and hence functionality, of the resulting "cluster assembled films."

This work was supported by the EPSRC and BNFL. S.J.C. and S.G.H. would like to thank the University of Birmingham and the EPSRC for support.

[1] For example, R. P. Andres, J. D. Bielefeld, J. I. Henderson, D. B. Janes, V. R. Kolagunta, C. P. Kubiak, W. J. Mahoney, and R. G. Osifchin, Science 273, 1690 (1996); J. Shi, S. Gider, K. Babcock, and R. D. Awschalom, Science 271, 937 (1996).

[2] For example, see Proceedings of ISSPIC 8 [Z. Phys. D 40 (1997)].

[3] R. E. Palmer, New Sci. 2070, 38 (1997).

[4] W. Harbich, S. Fedrigo, and J. Buttet, Z. Phys. D 26, 138 (1993).

[5] L. Bardotti, P. Jensen, A. Hoareau, M. Treilleux, and B. Cabaud, Phys. Rev. Lett. 74, 4694 (1995).

[6] G. L. Kellogg, Phys. Rev. Lett. 73, 1833 (1994).

[7] J.-M. Wen, S.-L. Chang, J. W. Burrnett, J. W. Evans, and P. A. Thiel, Phys. Rev. Lett. 73, 2591 (1994).

[8] P. Delotour, J. L. Barrat, and P. Jensen, Phys. Rev. Lett. 78, 4597 (1997).

[9] K. Bromann, C. Felix, H. Brune, W. Harbich, R. Monot, J. Buttet, and K. Kern, Science 274, 956 (1996).

[10] H.-P. Cheng and U. Landman, Science 260, 1304 (1993).

[11] H. Hsieh, R.S. Averback, H. Sellers, and C.P. Flynn, Phys. Rev. B 45, 4417 (1992).
[12] G. Vandoni, C. Félix, and C. Massobrio, Phys. Rev. B 54, 1553 (1996).

[13] G. Betz and W. Husinsky, Nucl. Instrum. Methods Phys. Res., Sect. B 122, 311 (1997).

[14] H. Haberland, M. Karrais, M. Mall, and Y. Thurner, J. Vac. Sci. Technol. A 10, 3266 (1992).

[15] S. J. Carroll, P. Weibel, B. von Issendorff, L. Kuipers, and R. E. Palmer, J. Phys. Condens. Matter 8, L617 (1996).

[16] S. J. Carroll, K. Seeger, and R. E. Palmer, Appl. Phys. Lett. 72, 305 (1998).

[17] G. M. Francis, L. Kuipers, J. R. A. Cleaver, and R.E. Palmer, J. Appl. Phys. 79, 2942 (1996).

[18] J. R. Hahn, H. Kang, S. Song, and I. C. Jeon, Phys. Rev. B 53, R1725 (1996).

[19] D. Marton, H. Bu, K. J. Boyd, S. S. Todorov, A. H. Al-Bayati, and J. W. Rabalais, Surf. Sci. 326, L489 (1995).

[20] K. Nordlund, J. Keinonen, and T. Mattila, Phys. Rev. Lett. 77, 699 (1996).

[21] Albert Gras-Martí, Roger Smith, Keith Beardmore, Jośe J. Jiménez-Rodríguez, Vladimir Konoplev, and Julio Ferrón, Comp. Mater. Sci. 3, 413 (1995).

[22] R.P. Webb, R. Smith, I. Chaakaraov, and K. Beardmore, Nucl. Instrum. Methods Phys. Res., Sect. B 112, 99 (1996).

[23] C. T. Riemann, S. Andersson, P.A. Bruhwiler, N. Martensson, and B.U.R. Sundqvist, Nucl. Instrum. Methods Phys. Res., Sect. B 122, 343 (1997).

[24] B. T. Kaiser, T. M. Bernhardt, and K. Rademann, Appl. Phys. A 66, S711-S714 (1998).

[25] D. W. Brenner, Phys. Rev. B 42, 9458 (1990).

[26] G. J. Ackland, G. Tichy, V. Vitek, and M. W. Finnis, Philos. Mag. A 56, 735 (1987).

[27] L. Bonačić-Koutecký, L. Češpiva, P. Fantucci, J. Pittner, and J. Koutecky̌, J. Chem. Phys. 100, 490 (1994).

[28] H. Rafii-Tabar and H. Kamiyama, Surf. Sci. 385, 187 (1997).

[29] S. G. Hall, M. B. Nielsen, A.W. Robinson, and R.E. Palmer, Rev. Sci. Instrum. 68, 3335 (1997).

[30] R. Smith and K. M. Beardmore, Thin Solid Films 272, 255 (1996).

[31] A. Wucher, A. D. Bekkerman, N. Kh. Dzhemilev, S. V. Verkhoturov, and I. V. Veryovkin, Nucl. Instrum. Methods Phys. Res., Sect. B 140, 311 (1998). 